\title{
A comparative study of the integer ambiguity validation procedures
}

\author{
J. Wang, M. P. Stewart, and M. Tsakiri \\ School of Spatial Sciences, Curtin University of Technology, GPO Box U1987, Perth, WA 6845, Australia
}

(Received December 20, 1999; Revised July 19, 2000; Accepted July 21, 2000)

\begin{abstract}
In GPS and GLONASS satellite-based positioning, the correct determination of the integer carrier phase ambiguities is the key to achieve precise positioning results. The process of determining the ambiguities, called ambiguity resolution, is usually separated into three steps: (a) estimating the float or real-valued ambiguities, (b) searching the best integer ambiguity set, (c) validating the best ambiguity set. Whilst the theory and methodology for ambiguity estimation and search are well documented, the ambiguity validation issue is still under investigation. Existing ambiguity validation procedures are based on various statistical assumptions and therefore, may have different performances in practical applications. In this paper, the major ambiguity validation procedures are analysed and their performances in both GPS and GLONASS ambiguity resolution are numerically compared. It is concluded that both the probability of estimating ambiguities correctly and confidence levels of the ambiguity discrimination tests are both important indicators of the reliability of ambiguity resolution.
\end{abstract}

\section{Introduction}

Satellite navigation systems GPS and GLONASS have been widely used in precise geodetic positioning and navigation. In the case of the GPS and GLONASS systems, two fundamental measurements can be decoded from the satellite signals, namely the pseudo-ranges and the carrier phases. Carrier phase measurements are much more precise than the pseudo-ranges, thus they are the primary measurements for precise positioning. However, the problem is that the carrier phase measurements are ambiguous, with the ambiguity (the integer number of signal wavelengths between satellite and antenna) being an unknown value a priori. Hence, the determination of the integer ambiguities, commonly referred to as ambiguity resolution (AR), is the most critical data analysis step for either the GPS or the GLONASS satellite positioning operation.

The concept of ambiguity resolution has its origins in Very Long Baseline Interferometry (VLBI) data processing (Rogers et al., 1978). From the very inception of GPS carrier phase positioning, the importance of ambiguity resolution for precise static relative positioning was emphasised (Counselman and Shapiro, 1979). With fixed integer ambiguities, the carrier phases can be used as unambiguous precise range measurements. Consequently, the geometry of the mathematical models of the carrier phases is strengthened. Therefore, ambiguity resolution can be also considered as part of the mathematical modelling of the carrier phases.

In ambiguity resolution, integer ambiguity parameters are initially treated as real (continuous) parameters. The realvalued ambiguity parameters, together with other unknown parameters such as the coordinates of the roving receiver can be estimated using least-squares or Kalman filtering algo-

Copy right (C) The Society of Geomagnetism and Earth, Planetary and Space Sciences (SGEPSS); The Seismological Society of Japan; The Volcanological Society of Japan; The Geodetic Society of Japan; The Japanese Society for Planetary Sciences. rithm. In the case of using least-squares, the so-called GaussMarkov model for linearised GPS/GLONASS (single- or double-differenced) measurements is written as

$$
\begin{aligned}
l & =A_{c} x_{c}+A_{k} x_{k}+v, \\
D & =\sigma^{2} Q=\sigma^{2} P^{-1},
\end{aligned}
$$

where

$l$ is the $n \times 1$ vector of the difference between the differenced GPS/GLONASS code and carrier phase measurements and their computed values, simply called the measurement vector, and $n$ is the number of the measurements;

$v$ is the $n \times 1$ vector of the random errors;

$x_{k}$ is the $m \times 1$ double differenced ambiguity parameter vector, and $m$ is the number of the ambiguity parameters;

$x_{c}$ is the $t \times 1$ vector of all other unknown parameters including position and other parameters of interest and $t$ is the number of all other unknowns except ambiguities;

$A_{k}$ is the design matrix for the ambiguity parameters;

$A_{c}$ is the design matrix for the other unknown parameters;

$D$ is the covariance matrix;

$Q$ is the cofactor matrix; 
$P$ is the weight matrix;

$\sigma^{2}$ is the a priori variance factor.

Based on the principle of least squares $\left(v^{T} P v=\right.$ minimum), the estimates of the unknowns $\hat{x}$ in Eq. (1) can be obtained by:

$$
\hat{x}=Q_{\hat{x}} A^{T} P l,
$$

with:

$$
Q_{\hat{x}}=\left(A^{T} P A\right)^{-1}
$$

where $\hat{x}=\left(\hat{x}_{c}, \hat{x}_{k}\right)^{T}$ and $A=\left(A_{c}, A_{k}\right) . Q_{\hat{x}}$ is the cofactor matrix of the estimated vector $\hat{x}$, which can be represented by the following partitioned matrices:

$$
Q_{\hat{x}}=\left[\begin{array}{cc}
Q_{\hat{x}_{c}} & Q_{\hat{x}_{c} \hat{x}_{k}} \\
Q_{\hat{x}_{k} \hat{x}_{c}} & Q_{\hat{x}_{k}}
\end{array}\right] .
$$

Furthermore, from Eqs. (1), (3) and (5), the least-squares residuals are obtained as:

$$
\hat{v}=l-A \hat{x}=Q_{\hat{v}} P l
$$

where $Q_{\hat{v}}=Q-A Q_{\hat{x}} A^{T}$ is the cofactor matrix of the residuals. With the estimated residual vector $\hat{v}$ and weight matrix $P$, the a posteriori variance cofactor can be estimated as:

$$
\hat{s}_{0}^{2}=\frac{\Omega_{0}}{f},
$$

where $\Omega_{0}=\hat{v}^{T} P \hat{v}=l^{T} P Q_{\hat{v}} P l=l^{T} P l-l^{T} P A \hat{x}$, and $f=n-m-t$.

The above float solution of the real-valued ambiguity estimates and their associated statistics is then used to construct a search window, which is assumed to contain the correct integer ambiguities. The process of searching all possible integer ambiguity combinations within the search window is then performed using a search criterion based on the minimisation of the quadratic form of the least-squares residuals. The best integer ambiguity combination that results in the minimum quadratic form of the least-squares residuals will be considered as the most likely (best) solution. Normally, the first two best ambiguity combinations are identified for validation purposes.

Ambiguity validation, however, is still a controversial problem under investigation. Existing ambiguity validation procedures are based on various statistical assumptions and therefore, may have different performances in practical applications. In this paper, these ambiguity validation procedures are analysed and their performances in both GPS and GLONASS ambiguity resolution are numerically compared.

\section{Ambiguity Validation Procedures}

Traditionally, ambiguity validation test procedures have been based on the so called $F$-ratio of the second minimum quadratic form of the least-squares residuals and the minimum quadratic form of the least-squares residuals (Counselman and Abbot, 1989). Recently, an ambiguity validation (discrimination) test procedure has been proposed
(Wang et al., 1998). This procedure is based on the ratio (called $W$-ratio) of the difference between the minimum and second minimum quadratic forms of the least-squares residuals and its standard deviation.

In the aforementioned two procedures, the fixed integer ambiguities are treated as non-random values. Another recent development focuses on the success probability of ambiguity resolution (or the probability of estimating the integer ambiguities correctly), in which integer ambiguities are considered as stochastic quantities (Teunissen, 1998).

Before discussing the results from a comparative study, a brief introduction of these ambiguity validation test procedures is given.

\section{1 $\quad F$-ratio test}

When the ambiguity parameter unknowns $x_{k}$ are assumed to be fixed to $K_{i}$, from a statistical point of view, it means that the following constraint equation:

$$
H x=K_{i}, \quad \text { with } \quad H=\left[0, E_{k}\right]
$$

is imposed to the original functional model represented by Eq. (1). In Eq. (8), $E_{k}$ is an $m \times m$ identity matrix. For such an ambiguity-fixed solution, the corresponding quadratic form of the residuals can be calculated with:

$$
\Omega_{i}=\Omega_{0}+R_{i}
$$

where

$$
R_{i}=\left(\hat{x}_{k}-K_{i}\right)^{T} Q_{\hat{x}_{k}}^{-1}\left(\hat{x}_{k}-K_{i}\right) .
$$

Assuming that the best and second best ambiguity combinations are $K_{m}$ and $K_{s}$, respectively, the corresponding quadratic forms are $\Omega_{m}$ and $\Omega_{s}$. The $F$-ratio is defined as

$$
F=\frac{\Omega_{s}}{\Omega_{m}} .
$$

The critical value of the $F$-ratio is often arbitrarily chosen as 2.0 (e.g. Euler and Landau, 1992). In this study, the $F$-ratio is approximately treated as a Fisher statistic for comparison.

\section{$2.2 W$-ratio test}

The $W$-ratio is defined as

$$
W=\frac{d}{\sqrt{\operatorname{Var}(d)}}
$$

where

$$
\begin{aligned}
& d=\Omega_{s}-\Omega_{m} \\
& \operatorname{Var}(d)=\delta^{2} Q_{d}
\end{aligned}
$$

$Q_{d}=4 \cdot\left(K_{s}-K_{m}\right)^{T} Q_{\hat{x}_{k}}^{-1}\left(K_{s}-K_{m}\right)$ is the cofactor of $d$, and $\delta^{2}$ is the so-called variance factor. Three different variance cofactors can be chosen (Wang et al., 1998). In this study, the a posteriori variance cofactor $\hat{s}_{0}^{2}$ is used. In this situation, the $W$-ratio has a Student's $t$ distribution.

\subsection{Success probability of ambiguity resolution}

Generally, it is extremely difficult to exactly evaluate the success probability of ambiguity resolution (Hassibi and Boyd, 1998; Teunissen, 1998). For the same data set, the success probability of ambiguity resolution may vary with 


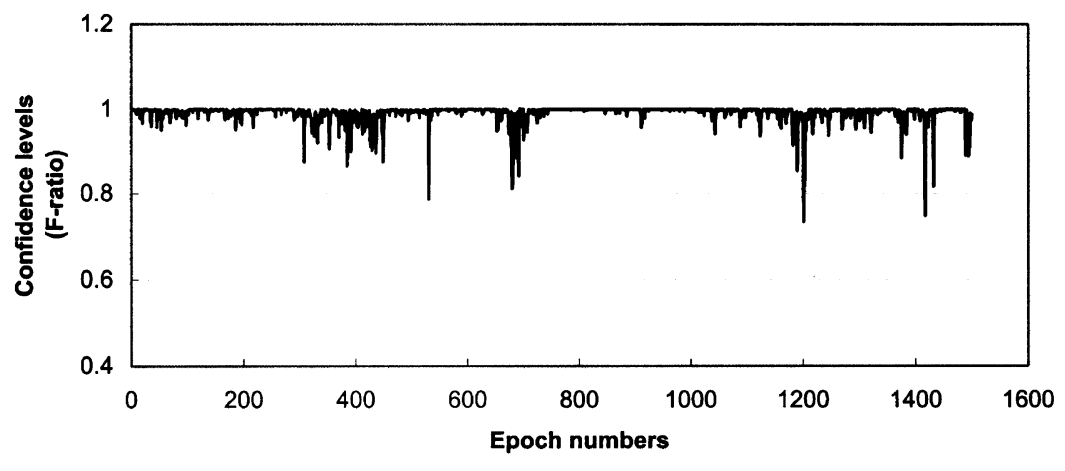

Fig. 1. Confidence levels ( $F$-ratio) for data set A.

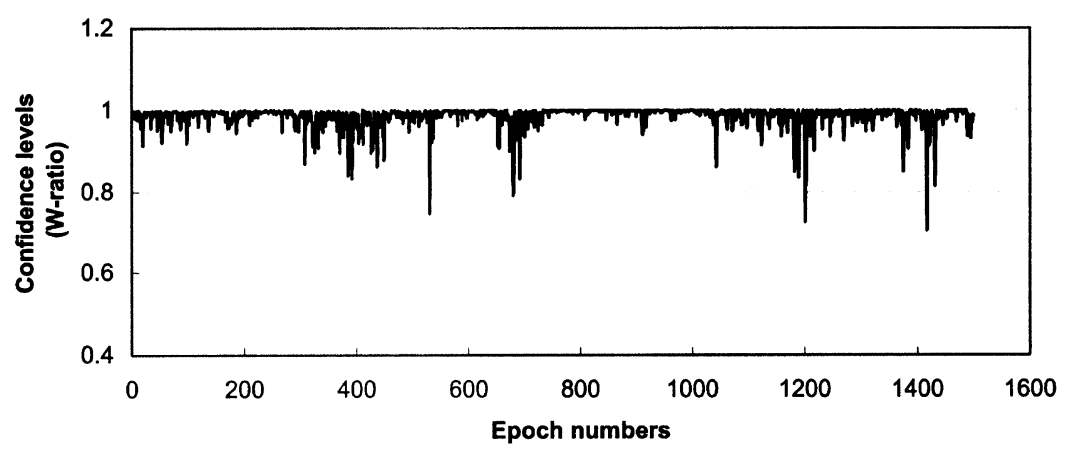

Fig. 2. Confidence levels ( $W$-ratio) for data set A.

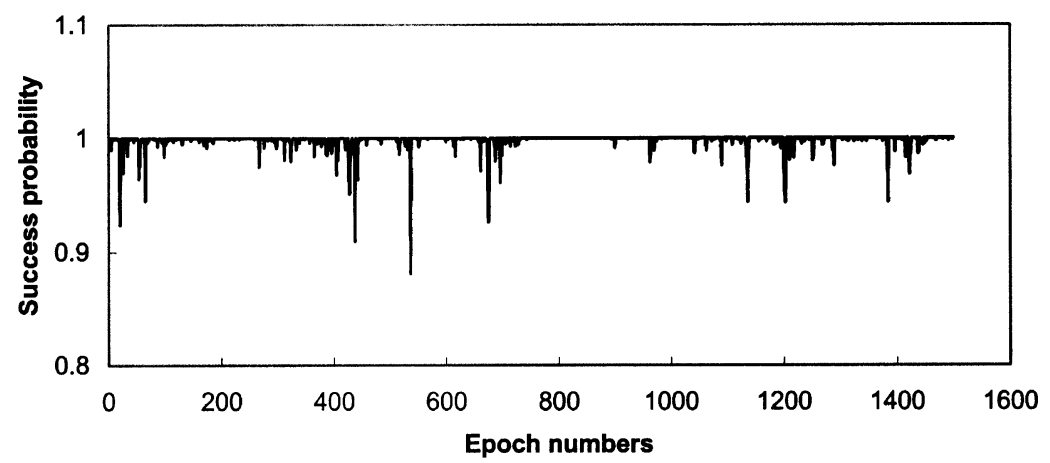

Fig. 3. Success probability for data set A.

the methods resolving the ambiguities. In the case of using the integer ambiguity bootstrapping method, the success probability can be exactly formulated as (Teunissen, 1998)

$$
P\left(K_{m}=a\right)=\prod_{i=1}^{m}\left(2 \Phi\left(\frac{1}{2 \sigma_{x_{i} \backslash I}}\right)-1\right) .
$$

Where

$a$ is the true but unknown integer ambiguities;

$\Phi(x)$ is the integral of standard normal distribution from minus infinity to $x$; $\sigma_{x_{i} \backslash I}$ is the conditional standard deviations of the estimated ambiguities.

To get more reliable ambiguity resolution results, the DD ambiguities are transformed using the LAMBDA method (Teunissen, 1993). Actually, like the DOP values for GPS/ GLONASS point positioning, the success probability can be evaluated at the planning stage of positioning. At this stage, the a priori stochastic model is used. If the estimated stochastic model is employed, the success probability calculated using Eq. (15) is an approximated value. 


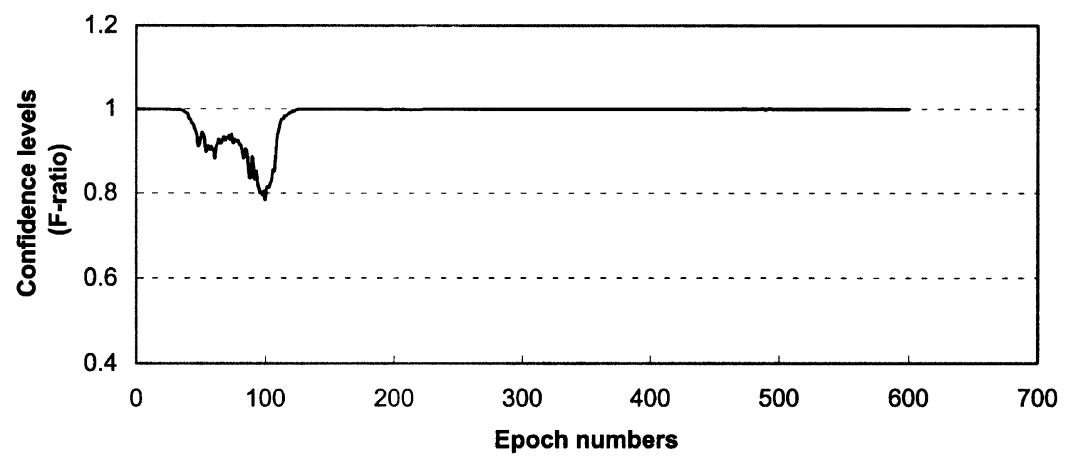

Fig. 4. Confidence levels ( $F$-ratio) for data set B.

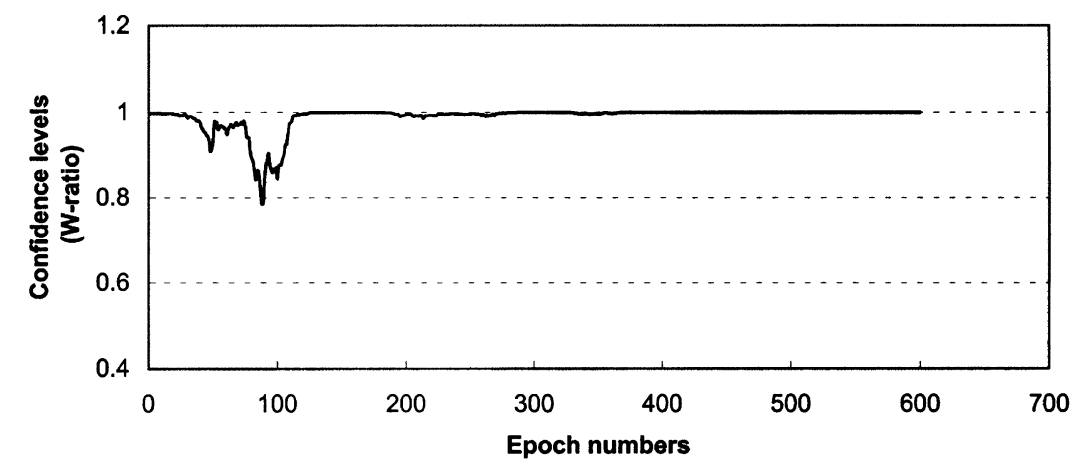

Fig. 5. Confidence Levels ( $W$-ratio) for data set B.

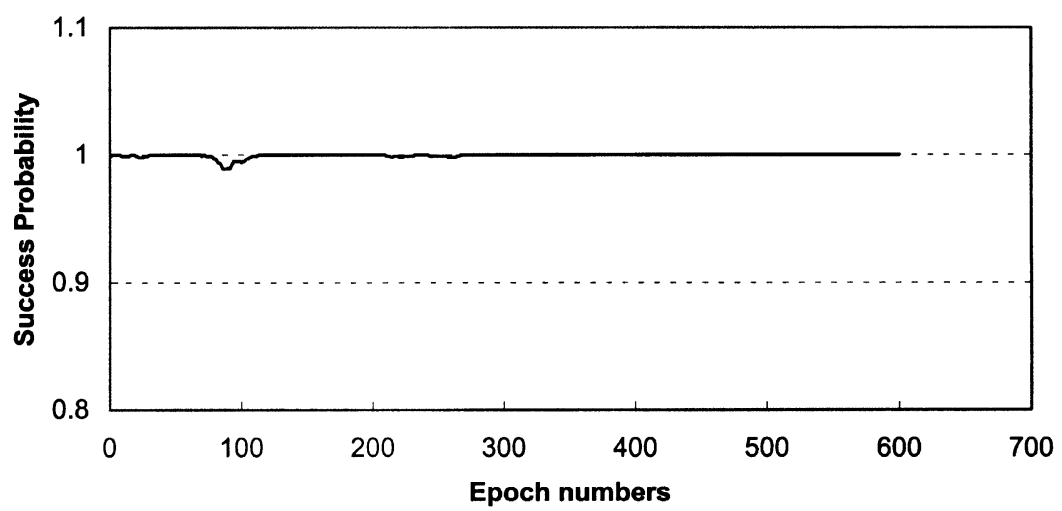

Fig. 6. Success probability for data set B.

\section{Testing Results and Analysis}

To compare the aforementioned ambiguity validation test procedures, both GPS dual-frequency data set and combined GPS/GLONASS single-frequency data set have been analysed.

\subsection{Description of the data sets}

Data set $A$ : the combined GPS/GLONASS data set was collected on a $1.2 \mathrm{~km}$ (static) baseline, on February 16, 1998, in Perth, Australia, using two Ashtech GG24 GPS/ GLONASS receivers. The data span was 25 minutes with the data interval of 1 second. During the whole session of observation, 7 GPS and 5 GLONASS satellites were tracked.

Data set B: the dual-frequency GPS data set was collected on a $9 \mathrm{~m}$ (static) baseline, on January 24, 1997, in Perth, Australia, using two Ashtech Z12 GPS receivers. The data span was 10 minutes with the data interval of 1 second. During the whole session of observation, 6 GPS satellites were tracked.

\subsection{Data processing}

All the data were processed epoch by epoch. The standard deviations for $\mathrm{L} 1$ and $\mathrm{L} 2$ code measurements are $1.0 \mathrm{~m}$, while the standard deviations for $\mathrm{L} 1$ and $\mathrm{L} 2$ carrier phases are 
$0.010 \mathrm{~m}$ and $0.012 \mathrm{~m}$, respectively. In ambiguity validation tests, the estimated variance factor was used.

Confidence levels (see Pearson and Hartley (1972) for their definitions) of the ambiguity validation tests using both the $F$ - and $W$-ratios are shown in Figs. 1, 2, 4 and 5. Figures 3 and 6 indicate the success probability values for the two test data sets.

\subsection{Discussions}

The above figures show that, overall, the confidence levels and the success probability values have a similar trend. This indicates that the compared ambiguity validation test procedures provide similar, but not necessary relevant statistics.

In some situations, there are big differences between the confidence levels and the success probability values. For example, in the case of data set B, significant discrepancy of the solutions between epochs 50 and 100 was noted. Actually, among these solutions, 5 best ambiguity combinations were incorrect, whilst the success probability values were very high (close to 1.0). In contrast, the confidence levels of the ambiguity validation tests using $W$ - or $F$-ratios were relatively much lower for these incorrect ambiguity combinations (see Figs. 4, 5 and 6). One possible reason for this is that the success probability, which is mainly dependent on the precision of ambiguities, is not sensitive to the model errors.

In GPS/GLONASS data processing, due to the existence of unmodelled systematic errors in measurements, the precision of estimated parameters may not always reflect their accuracy. Therefore, it is even likely that the best ambiguity combination is wrong. Actually, in some cases, it is extremely difficult to distinguish the correct ambiguity combination from wrong ones (Wang et al., 1998). An ambiguity discrimination test will function as a check on the reliability of ambiguity resolution.

\section{Concluding Remarks}

Ambiguity validation test is one of the critical steps in ambiguity resolution. This is the first effort to compare three different ambiguity validation procedures. From our investigations, the results of which have been presented in this paper, we can see that the confidence levels of the ambiguity discrimination tests based $F$ - and $W$-ratios are generally close to the success probability of ambiguity resolution.

However, ambiguity resolution with a high success prob- ability may end up with incorrect ambiguities due to various reasons, such as systematic errors in the measurements. The ambiguity discrimination test can be used as a second check on the reliability of the resolved integer ambiguities.

It is noted that the ambiguity discrimination test statistic ( $W$-ratio) is derived under the assumption that the fixed ambiguities are deterministic quantities. It is also of importance to make sure that the probability of correct integer ambiguity resolution is sufficiently close to one. Therefore, both the probability of estimating ambiguities correctly and confidence levels of the ambiguity discrimination tests are important indicators of the reliability of ambiguity resolution.

It should be pointed out that Eq. (15) is derived using a priori variance factor, which is mainly used in the stage of positioning design. However, in the stage of data processing, a posteriori (or estimated) variance factor may be used. In this situation, a rigorous formula for computing the success probability needs to be further investigated.

\section{References}

Counselman, C. C. and R. Abbot, Method of resolving radio phase ambiguity in satellite orbit determination, J. Geophys. Res., 94(B6), 7058-7064, 1989.

Counselman, C. C. and I. I. Shapiro, Miniature interferometric terminals for Earth surveying, Bulletin Géodésique, 53, 139-163, 1979.

Euler, H. J. and H. Landau, Fast GPS Ambiguity Resolution On-the-fly for Real-time Applications, Proceedings of 6th International Symposium on Satellite Positioning, Columbus, Ohio, 17-20 March, 650-659, 1992.

Hassibi, A. and S. Boyd, Integer parameter estimation in linear models with applications to GPS, IEEE Transactions on Signal Processing, 46, 2938-2952, 1998.

Pearson, E. S. and H. O. Hartley, Biometrika Tables for Statisticians (Volume II), pp. 53-66, Cambridge University Press, Cambridge, UK, 1972.

Rogers, A. E. E., C. A. Knight, H. F. Hinteregger, and A. R. Whitney, Geodesy by radio interferometry: Determination of a $1.24 \mathrm{~km}$ base line vector with $\sim 5 \mathrm{~mm}$ repeatability, J. Geophys. Res., 83(B1), 325-334, 1978

Teunissen, P. J. G., Least-Squares Estimation of the Integer GPS Ambiguities, Invited Lecture, Section IV, Theory and Methodology, IAG General Meeting, Beijing, China, August, 16 pp., 1993.

Teunissen, P. J. G., Success probability of integer GPS ambiguity rounding and bootstrapping, J. Geod., 72, 606-612, 1998.

Wang, J., M. Stewart, and M. Tsakiri, A discrimination test procedure for ambiguity resolution on-the-fly, J. Geod., 72, 644-653, 1998.

J. Wang (e-mail: JL.Wang@unsw.edu.au), M. P. Stewart, and M. Tsakiri 